\title{
Design and Simulation of Reflectarray Metasurfaces in the Visible Regime
}

\author{
SAlma Alrasheed ${ }^{1 *}$, Enzo Di FabrizIO ${ }^{1}$ \\ ${ }^{1}$ King Abdullah University of Science and Technology, PSE and BESE Divisions, Thuwal, 23955-6900, Kingdom of Saudi Arabia
}

*Corresponding author: salma.alrasheed@kaust.edu.sa

Received XX Month XXXX; revised XX Month, XXXX; accepted XX Month XXXX; posted XX Month XXXX (Doc. ID XXXXX); published XX Month XXXX

\begin{abstract}
Metasurfaces are new promising ultrathin materials that can enable many novel optical devices due to its ability to act as a discontinuity interface that introduces an abrupt change in amplitude, phase and sometimes the polarization of the incident light at the wavelength scale. Therefore they can function as flat optical elements. Here, we investigate the anomalous reflection of light for TM polarization for normal and oblique incidence in the visible regime. We propose gradient phase gap surface plasmon (GSP) metasurfaces that exhibit high conversion efficiency (up to $\sim 97 \%$ of total reflected light) to the anomalous reflection angle for blue, green and red wavelengths at normal and oblique incidence, and where light polarization is unchanged after the reflection.
\end{abstract}

OCIS codes: (250.5403) Plasmonics; (160.3918) Metamaterials; (110.5100) Phased-array imaging systems; (330.1690) Color.

http://dx.doi.org/10.1364/AO.99.099999

\section{INTRODUCTION}

Conventional optics modifies the wave front of light through propagation, where the change in phase gradually accumulates as the light propagates. If an abrupt phase change exists at the interface between two media, over a thickness of the scale of a wavelength, the normal laws of reflection and refraction no longer hold. Such phase shift can be created using a two dimensional array made of optical resonators with subwavelength separation and a phase response that is spatially dependent. This is because light scattered from optical resonators can introduce abrupt phase and sometimes polarization changes relative to the incident light. If the phase shift varies linearly with position, the incident plane wave remains intact resulting in anomalous reflection and refraction. The amount by which the incident plane wave is bent is proportional to the phase gradient [1-5].

Two dimensional arrays made of optical resonators with sub-wavelength periodicity are known as metasurfaces, and because of their thickness which is negligible compared to the incident wavelength (thickness $\sim \lambda / 20$ ), they can be considered near resonances of the constituents as a discontinuity interface that introduces an abrupt change in the amplitude, phase and sometimes polarization of the incident light at the wavelength scale. Therefore they can function as flat optical elements [1,611].

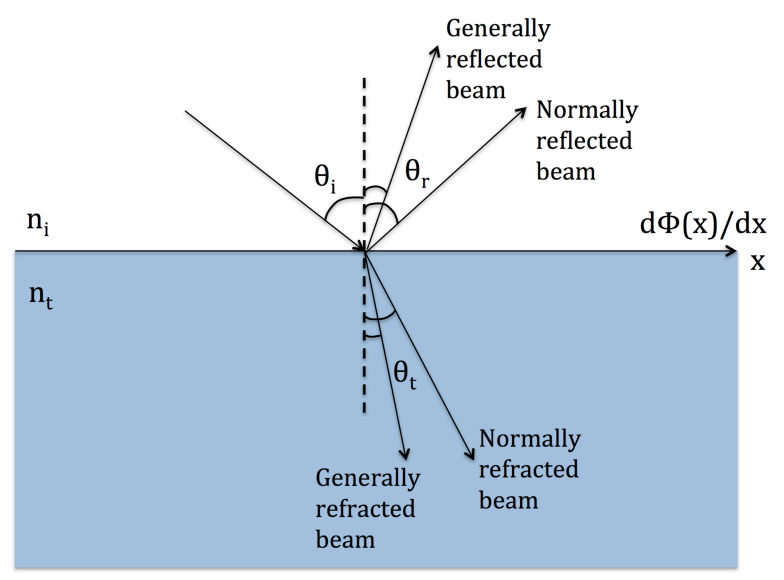

Fig. 1. Schematic representation of the generalized laws of reflection and refraction. At the interface, a sudden phase shift $\Phi(\mathrm{x})$ that linearly depends on $\mathrm{x}$ is introduced.

If the phase gradient along the interface between the two media, as in Fig. 1., is constant, then by using the conservation of the wavevector along the interface, or alternatively by using Fermat's principle, the resulting generalized laws of reflection and refraction are 


$$
\begin{aligned}
& \sin \left(\theta_{t}\right) n_{t}-\sin \left(\theta_{i}\right) n_{i}=\frac{1}{k_{o}} \frac{d \Phi}{d x} \\
& \sin \left(\theta_{r}\right)-\sin \left(\theta_{i}\right)=\frac{1}{k_{o} n_{i}} \frac{d \Phi}{d x}
\end{aligned}
$$

Where $k_{o}$ is the free space wavevector, $n_{i}$ and $n_{t}$ are the refractive indices of the two media, $d \Phi / d x$ is the phase gradient along the interface, and $\theta_{i}, \theta_{t}$ and $\theta_{r}$ are the incident, transmission and reflection angles respectively. The generalized laws of refraction and reflection can be shown to be equivalent to a blazed grating in that it eliminates all but one diffraction order, although the mechanism is different, since blazed gratings rely on gradual phase accumulation. Therefore, the metasurface can be considered as a flat blazed grating, where the anomalous angle of reflection equals one of the diffraction angles [12].

To achieve the full phase change of $2 \pi$, two different methods were used $[1,7,8,13-28]$. In the first approach, the scattered cross-polarized light relative to the incident light is manipulated using V-shaped nanoantennas, which demonstrates generalized reflection and refraction. However even in the case of negligible loss, the upper theoretical efficiency limit of this approach is $~ 25 \%[1,7,8]$. In the second approach, the metasurface is separated from a metal substrate by a dielectric spacer. Such configuration works only in reflection mode, but can have a high efficiency reaching $100 \%$ for negligible loss [2-4,29-41].

For the visible and NIR range using plasmonic metallic structures, anomalous reflection was demonstrated using silver and gold, but with low efficiencies in the blue and green wavelengths [30,26-28,39]. Other structures used for better efficiency are silver trapezoid shaped nanorods [29]. Our designs use both aluminum and silver and demonstrate high efficiency anomalous reflection and broad working bandwidth in the visible regime. Aluminum is a promising plasmonic material with a strong plasmonic response that spans much of the visible spectrum, and together with its low cost has gained increasing attention recently [40-42].

The basic element used in the second approach of metalbacked metasufraces is the metal-insulator-metal (MIM) resonator as shown in Fig. 2. Such resonator supports gapsurface plasmon (GSP) resonances, which are standing waves resonances resulting from forward and backward travelling slow SPPs in the gap between the metal strip and the metal substrate. This causes peaks in the scattering spectra at the resonance wavelength in addition to a local field enhancement in the gap. At the GSP resonance, the phase variation is that of a Lorentz-like oscillator, where it varies over the range of $\pi$ $[2,4,43-46]$.

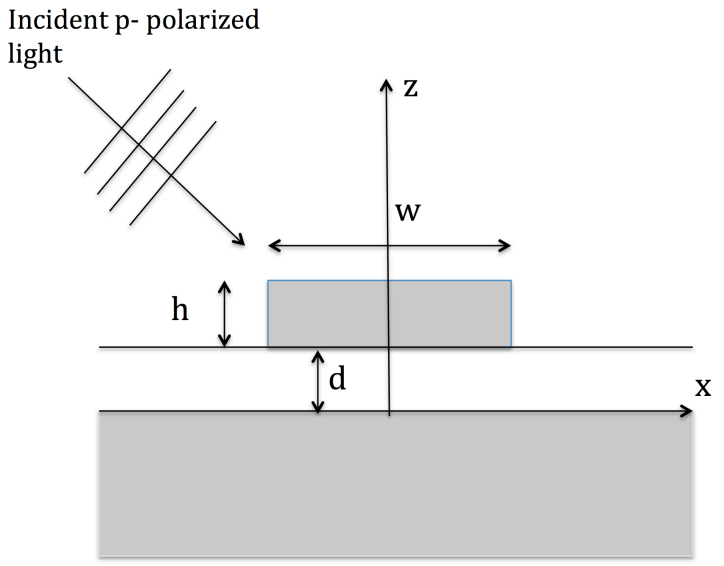

Fig. 2. A schematic representing the GSP resonator, where a TM polarized plane wave propagating in the $\mathrm{x}-\mathrm{Z}$ plane is scattered by a metal block of width $w$ and thickness $h$, separated from a metal surface by a dielectric layer of thickness $d$.

As the resonances are standing waves, their resonance can be calculated from the Fabry-Perot equation

$$
w k_{o} n^{\prime}=N \pi-\phi
$$

Where $w$ is the width of the block, $k_{o}$ is the vacuum wavenumber, $n^{\prime}$ and $n^{\prime \prime}$ are the real and imaginary parts of the slow GSP mode index $\left(\beta / k_{o}=n^{\prime}-i n^{\prime \prime}\right)$ respectively, $\phi$ is an additional phase shift acquired upon reflection of the SPP from the termination of the block and $\mathrm{N}$ is the order of the mode. This equation shows that a higher order GSP mode with the same wavelength is expected if $w$ is increased by $\Delta w=\left(\lambda_{o} / 2 n^{\prime}\right)[2-4,44]$.

The resonance peak broadens with increasing block's width $\mathrm{w}$, thickness $\mathrm{h}$ and gap size $\mathrm{d}$, this is because in all three cases the SPP becomes less strongly bound, resulting in weaker reflection at the block's boundaries thereby weaker resonances. GSP metasurfaces have the advantage of being much less sensitive to fabrication imperfections $[2,47]$.

Here we investigate the anomalous reflection of light for TM polarization for normal and oblique incidence in the visible regime. We propose gradient phase GSP metasurfaces in the visible regime that exhibit high conversion efficiency (up to $\sim 97 \%$ of total reflected light) to the anomalous reflection angle for blue, green and red wavelengths at normal and oblique incidence, and where light polarization is unchanged after the reflection. We use the commercial software (Lumerical) for the finite-difference time-domain method (FDTD) simulation. The 
building blocks of the metasurfrace are metal nanobricks with varying sizes arranged periodically. We use both $\mathrm{Al}$ and $\mathrm{Ag}$ for normal and oblique incidence respectively. Both metals exhibit better performance than $\mathrm{Au}$ particularly for shorter wavelengths, since for $\mathrm{Au}$, resonances below $520 \mathrm{~nm}$ are quenched due to interband transitions. For normal incident TM polarized light, we use aluminum metasurfaces, the reflected light is at $10^{\circ}$ to the normal. We design metasurfaces to demonstrate this effect at three different wavelengths of 445 $\mathrm{nm}, 530 \mathrm{~nm}$ and $650 \mathrm{~nm}$ (different array parameters for each wavelength). In the second configuration we show anomalous reflection of TM polarized light for an oblique incidence of $-63^{\circ}$, reflecting to an angle of $-10^{\circ}$ to the normal. For this scheme we use silver, and design three different metasurfaces operating at a wavelength of $430 \mathrm{~nm}, 520 \mathrm{~nm}$, and $620 \mathrm{~nm}$.

\section{STRUCTURE AND DESIGN}

A three-dimensional schematic of the $\mathrm{Al}$ and $\mathrm{Ag}$ metasurfaces used are shown in Fig. 3. (a) and (b) respectively. The metasurface consists of a periodic array of the supercell. The general design of the supercell for each of the $\mathrm{Al}$ and $\mathrm{Ag}$ array (consisting of more than one brick) is shown in Fig. 3. Note that the schematics shown are for the $\mathrm{Al}$ and $\mathrm{Ag}$ arrays for blue and green wavelengths respectively, however for other colors, the supercell generally has a similar design. For each color, the parameters of the supercell are given in Table. 1. and Table. 2. For the first example, the $\mathrm{Al}$ antennas of thickness of $50 \mathrm{~nm}$ are coupled with an optically thick Al substrate (500 nm thick) separated by a dielectric $\mathrm{SiO}_{2}(\mathrm{n}=1.45)$ spacer, which is also 50 $\mathrm{nm}$ thick. The array is in the $\mathrm{x}-\mathrm{y}$ plane and a TM plane wave source illuminates the metasurface at normal incidence along the z-axis. Due to the thickness of the Al substrate, the transmission is zero and the absorption $\mathrm{A}$ is found from $\mathrm{A}=1$ $\mathrm{T}-\mathrm{R}=1-\mathrm{R}$, where $\mathrm{R}$ represents the total reflectivity to all directions. For the second example, Ag antennas of thickness of $30 \mathrm{~nm}$ are coupled with an Ag (500 nm thick) substrate separated by a dielectric $\mathrm{SiO}_{2}(\mathrm{n}=1.45)$ spacer of thickness of $30 \mathrm{~nm}$.

For the Al array, a "super" unit cell consisting of 12 nanobricks arranged with a period from brick to brick center of $\Lambda_{\mathrm{x}}$ and a total cell length in the x-direction of $12\left(\Lambda_{\mathrm{x}}\right)$ and of a period of $\Lambda_{\mathrm{y}}$ in the $\mathrm{y}$-direction. For the Ag array, the super unit cell consists of 6 nanobricks, and the cell total length in $\mathrm{x}$ is $6\left(\Lambda_{\mathrm{x}}\right)$ and is $\Lambda_{\mathrm{y}}$ in the $\mathrm{y}$-direction. There are two main approaches for designing reflectarray metasurfaces, namely the phase-shift approach and the equivalence principle approach [48-50]. The equivalence principle approach is a more general approach using the exact solutions of Maxwell's equations. However for our purpose here, since the design is for a fixed incident angle, the phase-shift approach gives results that are in good agreement with the FDTD simulations. Using Eq. (2) together with $d \Phi / d x=m(2 \pi) n_{i} / d$, where $d \Phi / d x$ is the phase gradient along the interface, $m$ is the diffraction order, $n_{i}$ is the refractive index of the first medium and $d$ is the length of the supercell [12], The bricks dimensions are chosen such that they are separated by $60^{\circ}$ steps in their reflection phase for the $\mathrm{Al}$ array, while for the Ag array, all bricks have the same dimensions as they need to have the same phase of $360^{\circ}$ in order for light to be reflected into the -6 diffraction order. Note that this is equivalent to using a supercell consisting of only one brick with a period in $\mathrm{x}$ and $\mathrm{y}$ of $\Lambda_{\mathrm{x}}$ and $\Lambda_{\mathrm{y}}$ respectively, as then the $-10^{\circ}$ angle is equivalent to the -1 diffraction order. However, here we use 6 bricks to demonstrate the reflected plane wave more clearly. We use parameter sweeps for the brick dimensions $\mathrm{Lx}_{\mathrm{x}}$ and $\mathrm{L}_{\mathrm{y}}$ to find the optimum values for the design. Perfectly matched layer boundary conditions (PML) were used in the vertical (z) direction to eliminate unphysical scattering at the boundaries of the simulation region. The mesh accuracy is set to 3 , which corresponds to $\lambda / 14$ step size. The boundary conditions are periodic in both $\mathrm{x}$ and $\mathrm{y}$ directions to simulate an infinite array of the super cell. The complex refractive index of both $\mathrm{Ag}$ and $\mathrm{Al}$ is taken from the data of Palik $(0-2 \mu \mathrm{m})$ [51]. To measure the power reflected from the array, a 2D-z-normal (in the $x-y$ plane) frequency-domain power monitor is placed at a distance of $500 \mathrm{~nm} \pm 5 \mathrm{~nm}$ above the metasurface for all of the arrays. For the far-field polar plots, the far-field projection functions are used to propagate the reflected fields to a hemisphere of a radius of $1 \mathrm{~m}$ above the metasurface. The final images (polar plots) represent the field intensity on the hemisphere, as viewed from above the metasurface. Such feature utilizes the fields on the single surface of the frequency-domain power monitor, placed at $500 \mathrm{~nm} \pm 5 \mathrm{~nm}$ above the metasurface, and use them to calculate the far-fields at $1 \mathrm{~m}$ above that surface. For the phase of Ex plots, a 2D-y-normal (in the $x-z$ plane) frequency-domain profile monitor is placed at the center of the array. The parameters used for each color are given in Table 1. and Table 2.

Table 1. Al array unit cell bricks parameters $L_{x}$ and $L_{y}$ for the three colors, in addition to $\Lambda_{\mathrm{x}}$ and $\Lambda_{\mathrm{y}}$

\begin{tabular}{|c|c|c|c|c|c|c|c|c|c|}
\hline Color & $\begin{array}{l}\text { Wave- } \\
\text { length } \\
(\mathrm{nm})\end{array}$ & $\begin{array}{l}\mathbf{L}_{\mathrm{x} 1}{ }^{\prime} \\
\mathrm{L}_{\mathrm{y1}} \\
\text { (nm) } \\
\end{array}$ & $\begin{array}{l}L_{x x^{\prime}} \\
L_{y^{2}} \\
(n m) \\
\end{array}$ & $\begin{array}{l}\mathrm{L}_{x x^{\prime}} \\
\mathrm{L}_{\mathrm{y3}} \\
(\mathrm{nm}) \\
\end{array}$ & $\begin{array}{l}\mathrm{L}_{\mathrm{x}}{ }^{\prime} \\
\mathrm{L}_{\mathrm{y} \mathbf{y}^{\prime}} \\
\text { (nm) } \\
\end{array}$ & $\begin{array}{l}\mathrm{L}_{\mathrm{x}}{ }^{\prime} \\
\mathrm{L}_{\mathrm{y5}} \\
\text { (nm) } \\
\end{array}$ & $\begin{array}{l}\mathbf{L}_{x 6^{\prime}} \\
\mathrm{L}_{\mathrm{y6}} \\
(\mathrm{nm}) \\
\end{array}$ & $\begin{array}{l}\Lambda_{x} \\
(\mathrm{~nm})\end{array}$ & $\begin{array}{l}\Lambda_{y} \\
(\mathbf{n m})\end{array}$ \\
\hline Blue & 445 & $\begin{array}{l}20, \\
200\end{array}$ & $\begin{array}{l}58, \\
200\end{array}$ & $\begin{array}{l}80, \\
200\end{array}$ & $\begin{array}{l}138, \\
150\end{array}$ & $\begin{array}{l}170, \\
40\end{array}$ & $\begin{array}{l}220, \\
15\end{array}$ & 240 & 240 \\
\hline Green & 530 & $\begin{array}{l}20 \\
200\end{array}$ & $\begin{array}{l}76, \\
200\end{array}$ & $\begin{array}{l}96, \\
200\end{array}$ & $\begin{array}{l}120 \\
200\end{array}$ & $\begin{array}{l}144 \\
30\end{array}$ & $\begin{array}{l}204 \\
20\end{array}$ & 300 & 300 \\
\hline Red & 650 & $\begin{array}{l}43 \\
200\end{array}$ & $\begin{array}{l}98 \\
200\end{array}$ & $\begin{array}{l}122, \\
200\end{array}$ & $\begin{array}{l}156 \\
200\end{array}$ & $\begin{array}{l}220 \\
100\end{array}$ & $\begin{array}{l}250 \\
20\end{array}$ & 300 & 300 \\
\hline
\end{tabular}

Table 2. Ag array unit cell bricks parameters $L_{x}$ and $L_{y}$ for the three colors, in addition to $\Lambda_{\mathrm{x}}$ and $\Lambda_{\mathrm{y}}$

\begin{tabular}{lllll}
\hline Color & $\begin{array}{l}\text { Wave- } \\
\text { length } \\
(\mathbf{n m})\end{array}$ & $\begin{array}{l}\mathbf{L}_{\mathbf{x}^{\prime}} \\
\mathbf{L}_{\mathbf{y}} \\
(\mathbf{n m})\end{array}$ & $\begin{array}{l}\boldsymbol{\Lambda}_{\mathbf{x}} \\
(\mathbf{n m})\end{array}$ & $\begin{array}{l}\Lambda_{\mathbf{y}} \\
(\mathbf{n m})\end{array}$ \\
& & & \\
\hline Blue & 430 & $\begin{array}{l}260, \\
260\end{array}$ & 400 & 270 \\
Green & 520 & $\begin{array}{l}357, \\
360\end{array}$ & 500 & 370 \\
Red & 620 & $\begin{array}{l}500, \\
460\end{array}$ & 630 & 490 \\
\hline
\end{tabular}


(a)
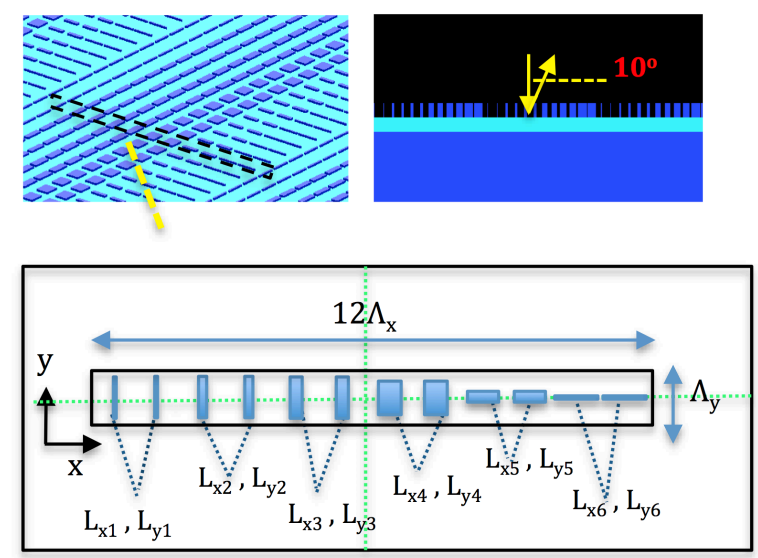

(b)

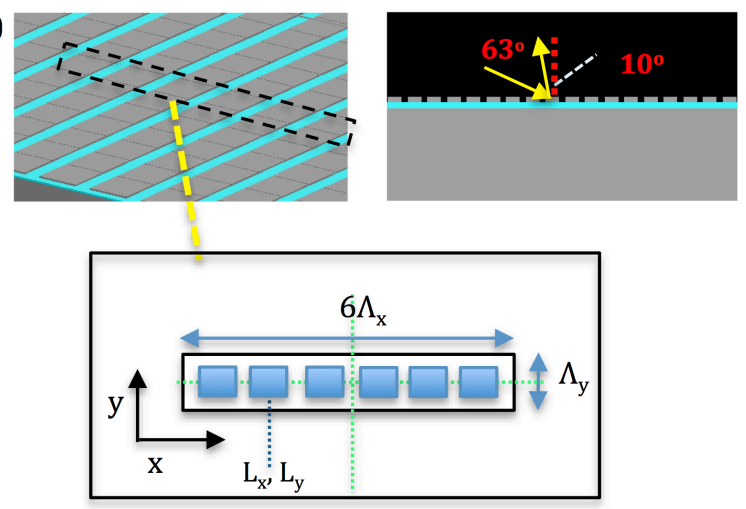

Fig. 3. (a) Schematics showing the designed Al array metasurface, where a single wavelength TM polarized light is normally incident on the array and reflected at $10^{\circ}$ to the normal. A single unit cell consists of an Al brick separated from a continuous $\mathrm{Al}$ substrate by a glass spacer, while the supercell consists of 12 bricks. The general design of the super cell of the array for each color is shown, where the dimensions used for each wavelengths of blue, green and red are shown in Table. 1. (a). (b) Schematics showing the designed Ag array metasurface, where a single wavelength TM polarized light is obliquely incident on the array at $-63^{\circ}$ to the normal and reflected at $-10^{\circ}$, a single unit cell consists of an Ag brick separated from a continuous Ag substrate by a glass spacer, while the supercell consists of 6 bricks. The general design of the supercell of the array used for the three colors is shown, where the dimensions used for each wavelengths of blue, green and red are shown in Table. 1. (b).

\section{RESULTS AND ANALYSIS}

For the Al arrays metasurfaces, TM polarized light is normally incident on the array and is reflected at an angle of $10^{\circ}$ to the normal (the first diffraction order). We designed a different array for each wavelength. Fig. 4. (a-c) shows the phase of $E_{x}$ for each $\mathrm{Al}$ array (in the $\mathrm{x}-\mathrm{z}$ plane) at each of the wavelengths of (a) $445 \mathrm{~nm}$ (b) $530 \mathrm{~nm}$ and (c) $650 \mathrm{~nm}$ respectively. Fig. 4. (d) shows the amount of source power reflected into the anomalous angle (the first diffraction order) for each of the $\mathrm{Al}$ arrays at the wavelengths of $445 \mathrm{~nm}, 530 \mathrm{~nm}$ and $650 \mathrm{~nm}$ represented by the blue, green and red lines respectively. Fig. 4. (e) shows the far-field intensity $|E|^{2}(\mathrm{v} / \mathrm{m})^{2}$ (on a hemisphere of a radius of $1 \mathrm{~m}$ above the metasurface) for the wavelength of $530 \mathrm{~nm}$. The far-field polar plots for the wavelengths of $445 \mathrm{~nm}$ and $650 \mathrm{~nm}$ are very similar. We calculated the total amount of reflected power relative to the source power for each array, and since there is no transmission, the rest of the source power is absorbed. The total reflected power amount is 73\%, $66.87 \%$ and $65.86 \%$ of the source power for each of the blue, green and red arrays respectively. For the blue array, since $66.4 \%$ of total source power is reflected into the first order (at an angle of $10^{\circ}$ to the normal), it follows that $91 \%$ of total reflected power goes into the anomalous mode. Similar calculations show that $90 \%$ and $96.6 \%$ of total reflected power goes into the anomalous reflection angle for each of the green and red arrays respectively. In addition, the resulting reflected beam is $p$ polarized for all arrays as the incident beam. Note that we used a pair of each element for the $\mathrm{Al}$ arrays to reduce the first order diffraction angle.

For the Ag arrays metasurfaces, TM polarized light is obliquely incident on the array at $-63^{\circ}$ to the normal and is reflected at $-10^{\circ}$ to the normal (diffraction order number of -6 ). As in the case for the $\mathrm{Al}$ arrays, we design a different array for each wavelength. Fig. 4. (f-h) shows the phase of $E_{x}$ for each $A g$ array (in the x-z plane) at each of the wavelengths of (a) 430 $\mathrm{nm}$ (b) $520 \mathrm{~nm}$ and (c) $620 \mathrm{~nm}$ respectively. Fig. 4. (i) shows the amount of source power reflected into the anomalous angle (the sixth diffraction order) for each of the Ag arrays at the wavelengths of $430 \mathrm{~nm}, 520 \mathrm{~nm}$ and $620 \mathrm{~nm}$ represented by the blue, green and red lines respectively. Fig. 4. (j) shows the far-field intensity $|E|^{2}(\mathrm{v} / \mathrm{m})^{2}$ (on a hemisphere of a radius of $1 \mathrm{~m}$ above the metasurface) for the wavelength of $520 \mathrm{~nm}$. The far-field polar plots for the wavelengths of $430 \mathrm{~nm}$ and $620 \mathrm{~nm}$ are very similar. We also calculated the total amount of reflected power relative to the source power for each array as $43.9 \%, 56.1 \%$ and $54.5 \%$ for the blue, green and red arrays respectively. While the amount of total reflected power going into the anomalous reflection mode is $96.6 \%, 91.2 \%$ and $64.5 \%$ for the blue, green and red arrays respectively. These results show the possibility to control light with high efficiency in the visible regime. 
(a)

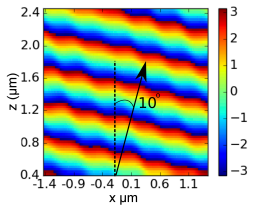

(d)

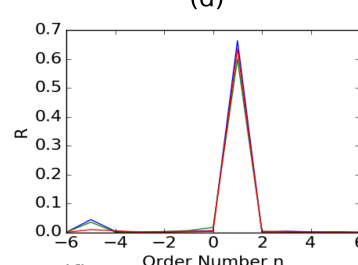

(f)

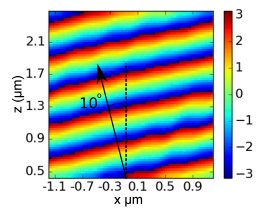

(i)

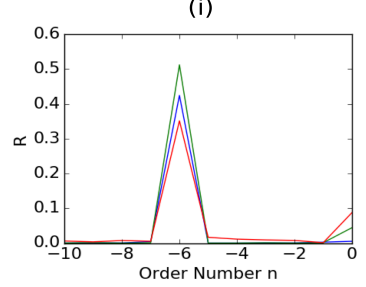

(b)
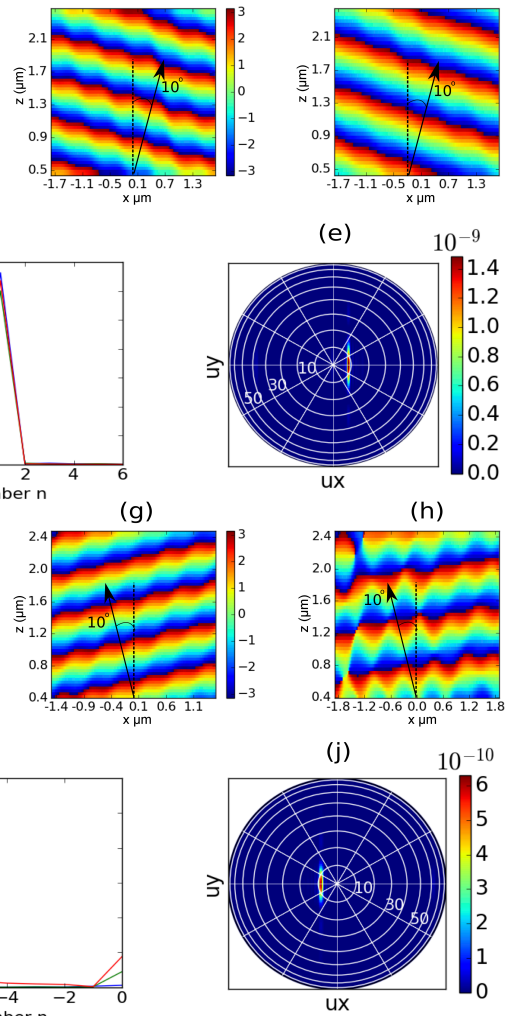

(e)

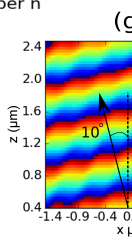

(c)
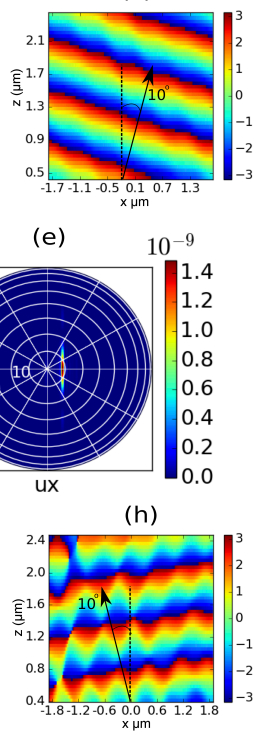

Fig. 4. $(a)(b)(c)$ The phase of $E_{x}$ for $A l$ array (in the $x-z$ plane) at a wavelength of $445 \mathrm{~nm}, 530 \mathrm{~nm}$ and $650 \mathrm{~nm}$ respectively. (d) Amount of source power reflected into each order for $\mathrm{Al}$ array at a wavelength of $445 \mathrm{~nm}, 530 \mathrm{~nm}$ and $650 \mathrm{~nm}$ (blue, green and red lines respectively). (e) The far-field intensity $|E|^{2}(\mathrm{v} / \mathrm{m})^{2}$ (on a hemisphere of a radius of $1 \mathrm{~m}$ above the metasurface) for the wavelength of $530 \mathrm{~nm}$. (f)(g)(h) The phase of $E_{x}$ for Ag array (in the $x-z$ plane) at a wavelength of $430 \mathrm{~nm}$, $520 \mathrm{~nm}$ and $620 \mathrm{~nm}$ respectively. (i) Amount of source power reflected into each order for Ag array at a wavelength of 430 $\mathrm{nm}, 520 \mathrm{~nm}$ and $620 \mathrm{~nm}$ (blue, green and red lines respectively). (j) the far-field intensity $|E|^{2}(\mathrm{v} / \mathrm{m})^{2}$ (on a hemisphere of a radius of $1 \mathrm{~m}$ above the metasurface) for the wavelength of $520 \mathrm{~nm}$.

Finally, we show how the efficiency and the angle of anomalous reflection evolves as the incident angle is changed for a given reflectarrray. For this purpose we choose the $\mathrm{Al}$ and $\mathrm{Ag}$ arrays used at the red $(650 \mathrm{~nm})$ and blue $(430 \mathrm{~nm})$ wavelengths respectively. The arrays at the other wavelengths show similar results. Using Eq. (2) together with $d \Phi / d x=m(2 \pi) n_{i} / d$, where $d \Phi / d x$ is the phase gradient along the interface, $m$ is the diffraction order, $n_{i}$ is the refractive index of the first medium and $d$ is the length of the supercell [12], the angle of anomalous reflection can be calculated for a given angle of incidence. For the $\mathrm{Al}$ array at the red wavelength of $650 \mathrm{~nm}$, Fig. 5. (a) shows the amount of source power reflected into the anomalous angle (here the first diffraction order), for different angles of incidence. For each color, $\left(\theta_{i}, \theta_{r}\right)$ is shown, where $\theta_{i}$ is the angle of incidence, and $\theta_{r}$ is the anomalous reflection angle (here the first diffraction order $(m=1)$ ). Note that the observed anomalous reflection angles (for $m=1$ ) from simulation match those that are calculated. As can be shown from Fig. 5. (a), most of the reflected light goes into the anomalous reflection angle (the first diffraction order of $m=1$ ) for incident angles up to the angle of $30^{\circ}$. The total amount of reflected power is then decreased for the incident angles of $40^{\circ}$ and $50^{\circ}$ with more light being distributed among different diffraction orders. However, at an incident angle of $60^{\circ}$, the total reflected power is increased again with most of the light being specularly reflected to the diffraction order $m=0$. Note that we referred to the reflected angle $\left(\theta_{r}=60^{\circ}\right)$ as $*$ in Fig. 5 (a), since here it corresponds to the zero diffraction order, and is not calculated using $\mathrm{m}=1$ as in the previous angles. Fig. 5 (b) shows the results obtained for the Ag array at the blue (430 $\mathrm{nm}$ ) wavelength where the amount of source power reflected into the anomalous angle (here the diffraction order $m=-6$ ) is shown for different angles of incidence. For a normal incidnet angle, the reflection is specular into the zero diffraction order $(\mathrm{m}=0)\left(\theta_{r}=0^{\circ}\right)$. Note that as in the case of the $\mathrm{Al}$ array, we referred to the reflected angle $\left(\theta_{r}=0^{\circ}\right)$ as ${ }^{*}$, since here it corresponds to the zero diffraction order, and is not calculated using $m=-6$ as for the other angles. As the incident angle increases, more light is reflected into the anomalous reflection angle $(m=-6)$ and less into the zero diffraction order (specular reflection) until it reaches a maximum at about $60^{\circ}$ where after that, the reflection into the zero order grows again. These results show that the arrays are flexible in handling different angles of incidence with a reasonable efficiency. 

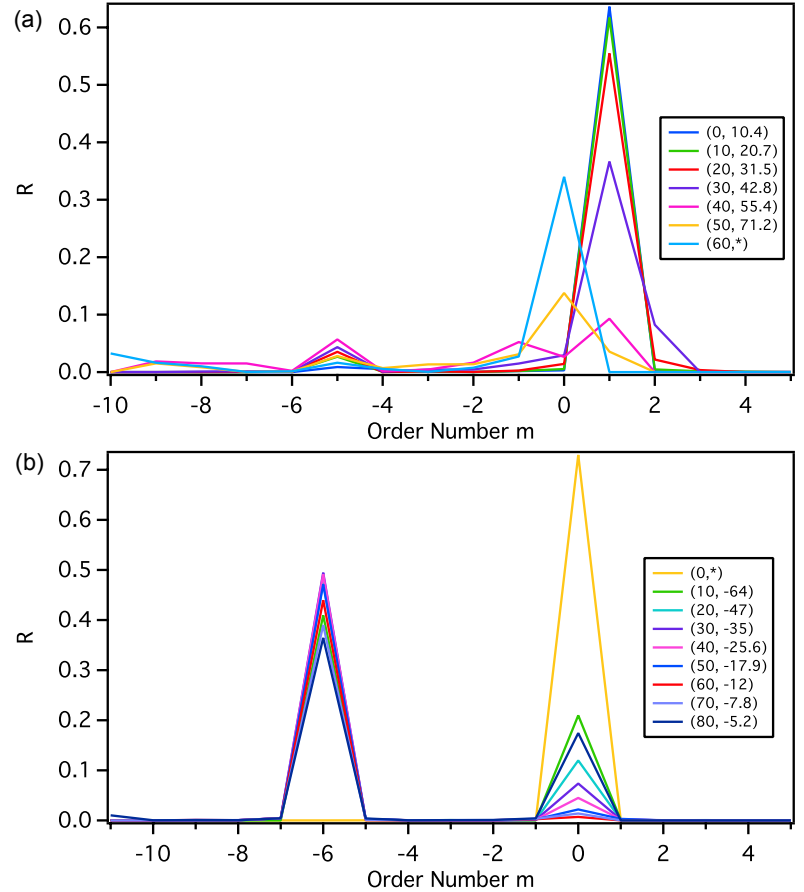

Fig. 5. (a) The amount of source power reflected into the anomalous angle (the first diffraction order), for different angles of incidence for the $\mathrm{Al}$ array at the wavelength of $650 \mathrm{~nm}$. For each color, $\left(\theta_{i}, \theta_{r}\right)$ is shown, where $\theta_{i}$ is the angle of incidence, and $\theta_{r}$ is the anomalous reflection angle (here the first diffraction order $(m=1)$ ). At an incident angle of $60^{\circ}$, most of the light undergo a specular reflection into the diffraction order $\mathrm{m}=0\left(\theta_{r}=60^{\circ}\right)$. Note that we referred to the reflected angle $\left(\theta_{r}=60^{\circ}\right)$ as ${ }^{*}$, since here it corresponds to the zero diffraction order, and is not calculated using $\mathrm{m}=1$ as in the previous angles. (b) The amount of source power reflected into the anomalous angle (here the diffraction order $\mathrm{m}=-6$ ), for different angles of incidence for the Ag array at the wavelength of $430 \mathrm{~nm}$. For a normal incidnet angle, the reflection is specular into the zero diffraction order $(\mathrm{m}=0)\left(\theta_{r}=0^{\circ}\right)$. Note that as in the case of the $\mathrm{Al}$ array, we referred to the reflected angle $\left(\theta_{r}=0^{\circ}\right)$ as *, since here it corresponds to the zero diffraction order, and is not calculated using $\mathrm{m}=-6$ as for the other angles.

\section{CONCLUSION}

We numerically studied the anomalous reflection of light for TM polarization for normal and oblique incidence in the visible regime. We proposed gradient phase GSP metasurfaces using Al and $\mathrm{Ag}$ for normal and oblique incidence respectively. We showed that high conversion efficiency (up to $\sim 97 \%$ of the total reflected light) to the anomalous reflection mode could be achieved. Such devices can be used as part of planar optical components with broad working bandwidth and can be integrated into photonic systems. In addition, since GSP metasurfaces are much less sensitive to fabrication imperfections [47], such devices have the advantage of being robust and scalable.

Funding. King Abdullah University of Science and Technology (OCRF2014-CRG3-62140384).

Acknowledgment. We thank SMILEs lab members at King Abdullah University of Science and Technology for the fruitful discussions and suggestions.

\section{References}

1. N.Yu, P. Genevet, M.A. Kats, F.Aieta, J.Tetienne, F.Capasso, Z.Gaburro, "Light Propagation with Phase Discontinuities: Generalized Laws of Reflection and Refraction," Science 334 (6054), 333-337 (2011).

2. A.Pors, O.Albrektsen, I.P. Radko and S.I. Bozhevolnyi, "Gap plasmon-based metasurfaces for total control of reflected light," Scientific Reports 3, 2155 (2013).

3. J.Jung and T.Søndergaard, "Gap plasmon-polariton nanoresonators: Scattering enhancement and launching of surface plasmon polaritons," Physical Review B 79, 035401 (2009).

4. A.Pors and S.I. Bozhevolnyi, "Plasmonic metasurfaces for efficient phase control in reflection," Optics Express 21 (22), 27438-27451 (2013).

5. M.Farmahini-Farahani and H.Mosallaei, "Birefringent reflectarray metasurface for beam engineering in infrared," Optics letters 38 (4), 462-464 (2013).

6. S. A.Maier, Plasmonics: Fundamentals and Applications (Springer 2007).

7. N.Yu and F.Capasso, "Flat optics with designer metasurfaces," Nature 13, 139-150 (2014).

8. N.Yu, P.Genevet, F.Aieta, M.A. Kats, R.Blanchard, G.Aoust, J. Tetienne, Z.Gaburro, and F.Capasso, "Flat Optics: Controlling Wavefronts with Optical Antenna Metasurfaces," IEEE Journal of Selected Topics in Quantum Electronics 19 (3), 4700423-4700423 (2013).

9. A. Nikolaenko, N. Papasimakis, A. Chipouline, F. De Angelis, E. Fabrizio, and N. Zheludev, "THz Bandwidth Optical Switching with Carbon Nanotube Metamaterial," Optics Express 20 (6), 6068-6079 (2012).

10. P. Lalanne and P. Chavel, "Metalenses at visible wavelengths: past, present, perspectives," https://arxiv.org/abs/1610.02507.

11. P. Lalanne, J. P. Hugonin, and J. C. Rodier, "Theory of Surface Plasmon Generation at Nanoslit Apertures," Phys. Rev. Lett. 95, 263902 (2005).

12. S.Larouche and D.R. Smith, "Reconciliation of generalized refraction with diffraction theory," Optics Letters 37 (12), 2391-2393 (2012).

13. M. Nielsen and S. Bozhevolnyi, "Gap Plasmon-Based Metasurfaces: Fundamentals and Applications," Proc. SPIE 9160, 916006 (2014). 
14. A. Pors, M. Nielsen, R. Eriksen, and S. Bozhevolnyi, "Broadband Focusing Flat Mirrors Based on Plasmonic Gradient Metasurfaces," Nano Lett. 13, 829-834 (2013).

15. Z. Li and K. Aydin, "Broadband metasurfaces for anomalous transmission and spectrum splitting at visible frequencies," EPJ Applied Metamaterials 2, (2015).

16. X. Ni, Z. Wong, M. Mrejen, Y. Wang, X. Zhang, "An ultrathin invisibility skin cloak for visible light," Science $\mathbf{3 4 9}$ (6254), 1310-1314 (2015).

17. X. Ni, S. Ishii, A. Kildishev and V. Shalaev, "Ultra-thin, planar, Babinet-inverted plasmonic metalenses," Light Sci. Appl. 2, e27 (2013).

18. Y. Xu, Y. Fu and H. Chen, "Planar gradient metamaterials," Nature Reviews Materials 1, 16067 (2016).

19. R. Devlin, M. Khorasaninejad, W. Chen, J. Oh, F. Capasso,"High efficiency dielectric metasurfaces at visible wavelengths," https://arxiv.org/abs/1603.02735.

20. S. Zhang, M. Kim, F. Aieta, A. She, T. Mansuripur, I. Gabay, M. Khorasaninejad, D. Rousso, X. Wang, M. Troccoli, N. Yu, F. Capasso, "High efficiency near diffraction-limited midinfrared flat lenses based on metasurface reflectarrays," Optics Express, 24 (16), 18024-18034 (2016).

21. K. Chong, I. Staude, A. James, J. Dominguez, S. Liu, S. Campione, G. Subramania, T. Luk, M. Decker, D. Neshev, I. Brener and Y. Kivshar, "Polarization-Independent Silicon Metadevices for Efficient Optical Wavefront Control," Nano Lett., 15 (8), 5369-5374 (2015).

22. Z. Li, L. Huang, K. Lu, Y. Sun, and L. Min, "Continuous metasurface for high-performance anomalous reflection," Applied Physics Express 7 (11), 112001 (2014).

23. X. Chen, L. Huang, H. Mühlenbernd, G. Li, B. Bai, Q. Tan, G. Jin, C. Qiu, S. Zhang and T. Zentgraf, "Dual-polarity plasmonic metalens for visible light," Nature Communications 3, 1198 (2012).

24. R. Devlin, M. Khorasaninejad, W. Chen, J. Oh, F. Capasso, "Broadband high-efficiency dielectric metasurfaces for the visible spectrum," Proceedings of the National Academy of Sciences 113, 10478 (2016).

25. F. Qin, L. Ding, L. Zhang, F. Monticone3, C. Chum, J. Deng, S. Mei, Y. Li, J. Teng, M. Hong, S. Zhang, A. Alù and C. Qiu, "Hybrid bilayer plasmonic metasurface efficiently manipulates visible light," Science Advances 2 (1), e1501168 (2016).

26. S. Sun, Q. He, S. Xiao, Q. Xu, X. Li and L. Zhou, "Gradientindex meta-surfaces as a bridge linking propagating waves and surface waves," Nature Materials 11 (5), 426431 (2012).

27. D. Wen, F. Yue, G. Li, G. Zheng, K. Chan, S. Chen, M. Chen, K. Li, P. Wong, K. Cheah, E. Pun, S. Zhang and X. Chen, "Helicity multiplexed broadband metasurface holograms," Nature Communications 6, 8241 (2015).

28. Guoxing Zheng, Holger Mühlenbernd, Mitchell Kenney, Guixin Li, Thomas Zentgraf \& Shuang Zhang, "Metasurface holograms reaching 80\% efficiency," Nature Nanotechnology 10, 308-312 (2015).

29. Z.Li, E.Palacios, S.Butun, and K.Aydin, "Visible-Frequency Metasurfaces for Broadband Anomalous Reflection and
High-Efficiency Spectrum Splitting," Nano Letters 15 (3), 1615-1621 (2015).

30. S.Sun, K.Yang, C.Wang, T.Juan, W.T.Chen, C.Y.Liao, Q.He, S.Xiao, W.Kung, G.Guo, L.Zhou, and D.Tsai, "HighEfficiency Broadband Anomalous Reflection by Gradient Meta- Surfaces," Nano Letters 12 (12), 6223-6229 (2012).

31. Y. Ho, B. Cheng, W. Hsu, C. Wang and D. Tsai, "Anomalous reflection from metasurfaces with gradient phase

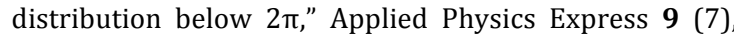
072502 (2016).

32. W. Hsu, P. Wu, J. Chen, T. Chen, Bo. Cheng, W. Chen, Y. Huang, Chun Y. Liao, G. Sun and D. Tsai, "Vertical splitring resonator based anomalous beam steering with high extinction ratio," Scientific Reports 5, 11226 (2015).

33. M. Kim, A. Wong, and G. Eleftheriades, "Optical Huygens' Metasurfaces with Independent Control of the Magnitude and Phase of the Local Reflection Coefficients," Physical Review X 4, 041042 (2014).

34. Y. Li, J. Zhang, S. Qu, J. Wang, Y. Pang, Z. Xu and A. Zhang, "Broadband unidirectional cloaks based on flat metasurface focusing lenses," Applied Physics 48 (33), (2015).

35. F. Ding, Z. Wang, S. He, V. Shalaev and A. Kildishey, "Broadband High-Efficiency Half-Wave Plate: A SupercellBased Plasmonic Metasurface Approach," ACS Nano 9 (4), 4111-4119 (2015).

36. M. Khorasaninejad, W. Chen, R. Devlin, J. Oh, A. Zhu, F. Capasso, "Metalenses at visible wavelengths: Diffractionlimited focusing and subwavelength resolution imaging," Science 352 (6290), 1190-1194 (2016).

37. S. Zhang, M. Kim, F. She, T. Gabay, M. Khorasaninejad, D. Rousso, X. Wang, M. Troccoli, N. Yu, F. Capasso, "High efficiency near diffraction-limited mid-infrared flat lenses based on metasurface reflectarrays," Optics Express 24 (16), 18024-18034 (2016).

38. S. Byrnes, A. Lenef, F. Aieta, and F. Capasso, "Designing large, high-efficiency, high-numerical-aperture, transmissive meta-lenses for visible light," Optics Express 24 (5), 5110-5124 (2016).

39. W. Chen, K. Yang, C. Wang, Y. Huang, G. Sun, I. Chiang, C. Liao, W. Hsu, H. Lin, S. Sun, L. Zhou, A. Liu and D. Tsai, "High-Efficiency Broadband Meta-Hologram with Polarization-Controlled Dual Images," Nano Lett. 14 (1), 225-230 (2014)

40. M. Knight, Nicholas. King, L. Liu, Henry O. Everitt, P. Nordlander and N. Halas, "Aluminum for Plasmonics," ACS Nano 8 (1), 834-840 (2014).

41. A. Moscatelli, "Plasmonics: The aluminium rush," Nature Nanotechnology 7, 778 (2012).

42. J.Olson, A.Manjavacas, .Liu, . Chang, B.Foerster, N.S. King, M.W. Knight, P.Nordlander, N.J. Halas, and S.Link, "Vivid, full-color aluminum plasmonic pixels," PNAS 11 (40), 14348-14353 (2014).

43. A.Pors and S.I. Bozhevolnyi, "Plasmonic metasurfaces for efficient phase control in reflection," Optics Express 21 (22), 27438-27451 (2013). 
44. T. Søndergaard and S. I. Bozhevolnyi, "Strip and gap plasmon polariton optical resonators," phys. stat. sol. (b) 245, 9 (1): 9-91 (2008).

45. Anders Pors and Sergey I. Bozhevolnyi, "Gap plasmonbased phase-amplitude metasurfaces: material constraints," Optical Materials Express 5 (11), 2448-2458 (2015).

46. Y. Lu, W. Dong, Z. Chen, A. Pors, Z. Wang and S. Bozhevolnyi, "Gap-plasmon based broadband absorbers for enhanced hot-electron and photocurrent generation," Scientific Reports 6, 30650 (2016).

47. Felipe Bernal Arango, Rutger Thijssen, Benjamin Brenny, Toon Coenen and A. Femius Koenderink, "Robustness of plasmon phased array nanoantennas to disorder," Scientific Reports 5, 10911 (2015).

48. C. Pfeiffer and A. Grbic, "Metamaterial Huygens' Surfaces: Tailoring Wave Fronts with Reflectionless Sheets," Phys. Rev. Lett. 110, 197401 (2013).

49. A. Epstein and G. Eleftheriades, "Huygens' metasurfaces via the equivalence principle: design and applications," Journal of the Optical Society of America B 33, A-31 (2016).

50. N. Estakhri and A. Alu, "Recent Progress in Gradient Metasurfaces," Journal of the Optical Society of America B 33, A-21 (2016).

51. E.D. Palik, Handbook of Optical Constants of Solids (Academic Press 1985). 